\title{
The Principles of Starch Gelatinization and Retrogradation*
}

\author{
Masakuni Tako $^{1,2 \#}$, Yukihiro Tamaki ${ }^{1}$, Takeshi Teruya ${ }^{1}$, Yasuhito Takeda ${ }^{3}$ \\ ${ }^{1}$ Department of Subtropical Bioscience and Biotechnology, University of the Ryukyus, Nishihara, Japan; ${ }^{2}$ Health and Longevity Re- \\ search Laboratory, Integrated Innovation Research Center, University of the Ryukyus, Nishihara, Japan; ${ }^{3}$ Department of Biochemical \\ Science and Technology, Kagoshima University, Kagoshima, Japan. \\ Email: \#tako@eve.u-ryukyu.ac.jp
}

Received November $29^{\text {th }}, 2013$; revised December $29^{\text {th }}, 2013$; accepted January $6^{\text {th }}, 2014$

Copyright (c) 2014 Masakuni Tako et al. This is an open access article distributed under the Creative Commons Attribution License, which permits unrestricted use, distribution, and reproduction in any medium, provided the original work is properly cited. In accordance of the Creative Commons Attribution License all Copyrights @ 2014 are reserved for SCIRP and the owner of the intellectual property Masakuni Tako et al. All Copyright (C) 2014 are guarded by law and by SCIRP as a guardian.

\section{ABSTRACT}

The polysaccharides, such as $\boldsymbol{\kappa}$-carrageenan, $\boldsymbol{t}$-carrageenan, agarose (agar), gellan gum, amylose, curdlan, alginate, and deacetylated rhamsan gum, in water changed into an ice-like structure with hydrogen bonding between polymer and water molecules, and between water-water molecules even at a concentration range of $0.1 \%-1.0 \%$ $(\mathrm{W} / \mathrm{V})$ at room temperature, resulting in gelation. Such dramatic changes from liquid into gels have been understood at the molecular level in principles. In this review, we describe the structure-function relationship of starch on the view point of rheological aspects and discuss gelatinization and retrogradation mechanism including water molecules at molecular level. The starch molecules (amylose and amylopectin) play a dominant role in the center of the tetrahedral cavities occupied by water molecules, and the arrangement is partially similar to a tetrahedral structure in a gelatinization process. The arrangement should lead to a cooperative effect stabilizing extended regions of ice-like water with hydrogen bonding on the surface of the polymer molecules, where hemiacetal oxygen and hydroxyl groups might participate in hydrogen bonding with water molecules. Thus, a more extended ice-like hydrogen bonding within water molecules might be achieved in a retrogradation process. Though many investigations not only include starch gelatinization and retrogradaion, but also the gelling properties of the polysaccharides have been undertaken to elucidate the structure-function relationship, no other researchers have established mechanism at the molecular level. There is reasonable consistency in our investigations.

\section{KEYWORDS}

Principles; Starch; Amylose; Amylopectin; Hydrogen Bonding; Gelatinization and Retrogradation Mechanism

\section{Introduction}

Starch is one of the most important polysaccharides and is a major component of many food plants such as wheat, barley, rice, corn, potato, sweet potato and cassava. Starch is used in food, cosmetics, paper, textile, and certain industries, as adhesive, thickening, stabilizing, stiffening, and gelling (pasting) agents. Starch consists of amylose

*Presented at the 26th International Carbohydrate Symposium, July 2227, 2012, Madrid, Spain and the 31st Annual Meeting of Japanese Society of Carbohydrate Research, September 17-20, 2012, Kagoshima, Japan.

"Corresponding author. and branched amylopectin molecules in molar ratios of $15 \%$ - $25 \%$ and $85 \%$ - $75 \%$, respectively.

We review the structure-function relationship of polysaccharides and starch on the view point of rheological aspects and discuss their gelation, gelatinization and retrogradation characteristics including water molecules at molecular level.

\section{Gelation Mechanism of Polysaccharides}

Polysaccharides that are produced commercially from seaweeds, plants, animals and micro-organisms have been used in food, cosmetic, paper, textile, oil, pharmaceutical, 
medical, and other industries as viscous enhancing, water-holding, emulsifying, oil-drilling, coating, encapsulating, protective, gelling and other agents. Specifically, for several decades attention has been directed to polysaccharide gels. Therefore, we have previously investigated the gelation mechanism of polysaccharides in aqueous solutions at a molecular level using a rheogoniometer.

In the course of the rheological study, we proposed gelation mechanism for $\kappa$-carrageenan [1,2], $l$-carrageenan [3], agarose (agar) [4], gellan gum [5], amylose [6,7], curdlan [8], alginate [9], deacetylated rhamsan gum [10], and native gellan gum [11] at the molecular level.

We also discussed the molecular origin for the thermal stability of non-gelling polysaccharides, such as welan [12,13], rhamsan [13,14], S-657 gum [15], S-88 gum [16] and schizophyllan [17]. The structure of these polysaccharides is similar to those of the gelling polysaccharides, gellan and curdlan. The thermal stability of the polysaccharides as described above has been attributed to intramolecular associations, where methyl and hydroxyl groups, and hemiacetal oxygen atom contributed.

We observed that there are some basic rules in the gel-formation processes of polysaccharides [18]. The gelation occurs because of the formation of intra- and inter-molecular associations, where hemiacetal oxygen, hydroxyl or methyl groups of the sugar residues of the polysaccharides contribute to hydrogen bonding or van der Waals forces of attraction. The sulfuric acid and carboxyl groups of the sugar residues of certain acidic polysaccharides, e.g., $\kappa$-carrageenan, $\boldsymbol{l}$-carrageenan, gellan gum, alginate, deacetylated rhamsan gum, and native gellan gum, also participated in intra- and/or inter-molecular associations through univalent or divalent cations that have ionic bonding or electrostatic forces of attraction. Specifically, the gel-formation of $\kappa$-carrageenan is specific for the large univalent $\mathrm{K}^{+}, \mathrm{Rb}^{+}, \mathrm{Cs}^{+}$, cations but not for the small $\mathrm{Li}^{+}$ or $\mathrm{Na}^{+}$cations, as illustrated in Figure 1. The cationspecific intramolecular bridge, which was preferentially placed to make polysaccharide molecule rigid, was the first demonstration of this effect at a molecular level [1]. An intermolecular association occurs between sulfuric acid groups on different molecules with electrostatic forces of attraction, as presented in Figure 2 [2]. These results suggest that the polysaccharide could have the application of removing the large radioactive $\mathrm{Cs}^{+}$cations in the environments polluted by the Fukushima Daiichi Nuclear Power Station in Japan.

\section{Co-Gelation Mechanism of Xanthan and Galactomannan, and Konjac Glucomannan}

Xanthan gum that is produced on a commercial scale using Xanthomonas campestris has curious rheological properties [19], viscosity and dynamic viscoelasticity of which show sigmoid curves during increase in tempera-

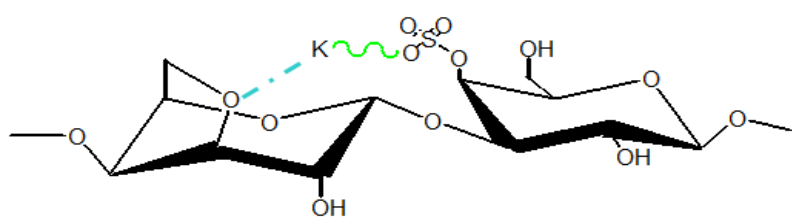

Figure 1. Intramolecular cation selective bridge in $\boldsymbol{\kappa}$-carrageenan in aqueous solution at low temperature: ( n ) ionic bonding and (- $-\ldots$ ) electrostatic forces of attraction. The large cations $\left(\mathrm{K}^{+} 275 \mathrm{pm}\right.$ in van der Waals rad., $\mathrm{Rb}^{+} 303 \mathrm{pm}$ and $\mathrm{Cs}^{+} 343 \mathrm{pm}$ are available to build up the bridge, but the small cations $\left(\mathrm{Li}^{+} 182 \mathrm{pm}\right.$ and $\left.\mathrm{Na}^{+} 227 \mathrm{pm}\right)$ are not.

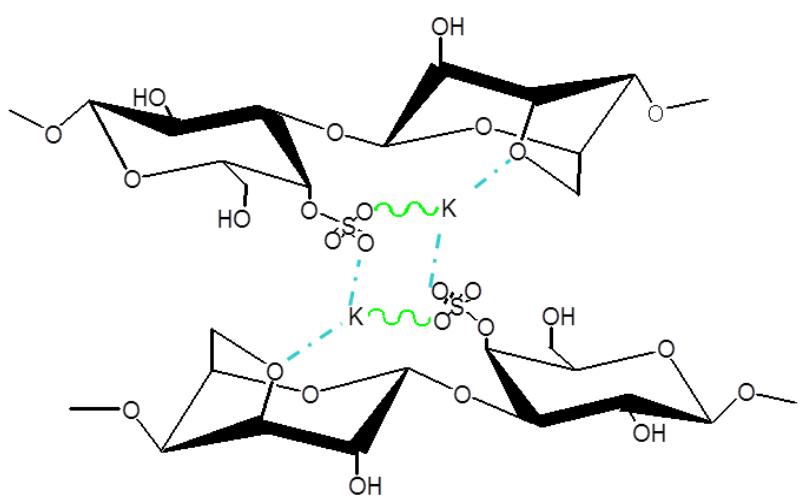

Figure 2. Gelation mechanism of $\boldsymbol{\kappa}$-carrageenan: (wn) ionic bonding and ( $-\ldots-\infty$ ) electrostatic forces of attraction. The model corresponds to a double stranded helix.

ture. The primary structure of xanthan is a 1,4 -linked $\beta$ D-glucan backbone (as in cellulose), C-3 substituted by a charged trisaccharide side-chain [20]. The internal mannose side-chain is substituted at C-6 with an acetyl group. About half or two-thirds of the terminal mannose residues bear a pyruvic acid. We discussed the molecular origin of its rheological characteristics [19,21-25] and proposed intramolecular associations in which the methyl group of the acetate substituted at the C-6 of D-mannopyranosyl residue on inner trisaccharide side-chains and the $\mathrm{OH}-3$ of D-glucopyranosyl residues on main-chain contributed to van der Waals forces of attraction and hydrogen bonding (Figure 3).

Furthermore, we discussed the molecular origin of the rheological characteristics for a mixed solution of xanthan and galactomannnans (locust-bean gum [26-28], guar gum [29], tara-bean gum [30], Leucaena gum [31], Leonix gum [32]), and konjac glucomannan [33,34]. A weak intermolecular interaction was observed in a mixture of high galactose content guar gum (33\%) or Leucaena gum $(40 \%)$. The results indicated that the weak interaction was due to the presence of side-chains of the galactomannan molecules. These side-chains prevented the insertion of the charged trisaccharide side-chains of the xanthan molecules into the galactomannan molecules. A least elastic modulus (G') was observed in the pres- 


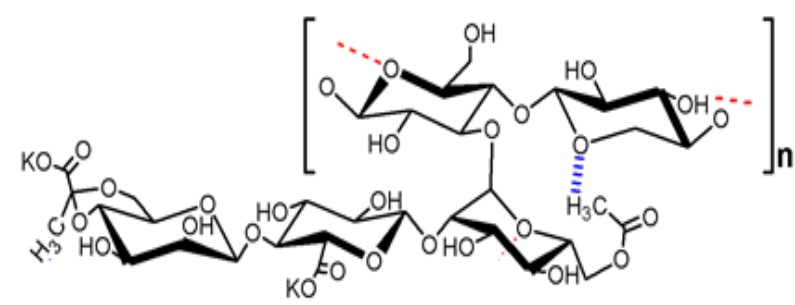

Figure 3. Intramolecular associations in xanthan molecules. The dotted lines represent hydrogen bonding and broken line van der Waals forces of attraction. The xanthan molecule may keep an ordered, rod-like, rigid, and less extended conformation owing to the formation of the intramolecular associations in aqueous solution.

ence of $\mathrm{Ca}^{+2}$ cations. Thus we proposed a co-gelation mechanism at the molecular level (Figure 4), in which the charged trisaccharide side-chains of the xanthan molecules take part in the gelation. The hemiacetal oxygen atom of inner D-mannosyl residue and pyruvate methyl group substituted at terminal D-mannosyl residue might take part in the interaction with the $\mathrm{OH}-2$ which oriented at axial configuration of D-mannosyl residues of the galactomannan main-chain. The co-gelation mechanism of xanthan and galactomannan (Figure 4) has given us to analyze a gelatinization and retrogradation mechanism of starch at molecular level which present later on.

More strong co-gelation than that of galactomannan (locust-bean gum) occurred in a mixture solution of xanthan and konjac glucomannan $[34,35]$ due to free from side-chains on the latter molecules.

The D-mannose-specific interaction between the extracellular bacterial polysaccharide xanthan and typical galactomannan components of the plant cell wall suggest a role in the host-pathogen relationship because Xanthomonas campestris is one of the plant pathogen bacteria.

\section{Principle of Polysaccharide Gels}

The $\mathrm{H}_{2} \mathrm{O}$ molecule can participate in four hydrogen bonds, two of them involving the two hydrogens of the molecule and the lone pair of electrons of the oxygen and hydrogens of two neighboring molecules. This tetrahedrally directed bonding is involved in the crystal structure of ice, which is the same as the arrangement of the gelling waters in polysaccharide solution.

At a minimum concentration of $0.1 \%-1.0 \%(\mathrm{~W} / \mathrm{V})$ in water $(99.9 \%$ - 99.0\%), the gelling polysaccharides mentioned above changed into an ice-like structure with the formation of hydrogen bonding between the polysaccharides and water, and between water molecules, which subsequently resulting in gelation even at room temperature. Such dramatic changes from liquid into gels have generally been understood at the molecular level [18].

We previously demonstrated the role of polysaccharides (e.g., agarose) in gel-formation processes in water
[18]. Agarose consists of a copolymer with alternating $O$-3-linked $\beta$-D-galactopyranosyl and $O$-4-linked 3,6anhydro- $\alpha$-L-galactopyranosyl residues. The structure is similar to that of $\kappa$-carrageenan and $l$-carrageenan, except for the sulfate content and l-configuration. As illustrated in Figure 5, intramolecular hydrogen bonding occurs between $\mathrm{OH}-4$ which oriented at axial configuration of the $\beta$-D-galactopyranosyl and the adjacent hemiacetal oxygen atom of the anhydro- $\alpha$-L-galacctopyranosyl residues [4]. The anhydro-L-galactopyranosyl residue is a cage-like sugar that contributes by stabilizing the proposed intramolecular hydrogen bonding, even at high temperature $>60^{\circ} \mathrm{C}$. Intermolecular hydrogen bonding occurs between the ring O-3,6-atom and the $\mathrm{OH}-2$ which oriented at axial configuration of anhydro-L-galactopyranosyl residues on different molecules. The association results in cage effect, which leads to the lowest energy state of electrons of the lone pairs of ring oxygen atoms of the anhydro-L-galactopuranosyl residues. The conformation adopts a tetrahedral distribution, and therefore, attracts not only each other but also water molecules due to hydrogen bonding [18]. The intra- and inter-molecular hydrogen bonding of agarose molecules results in

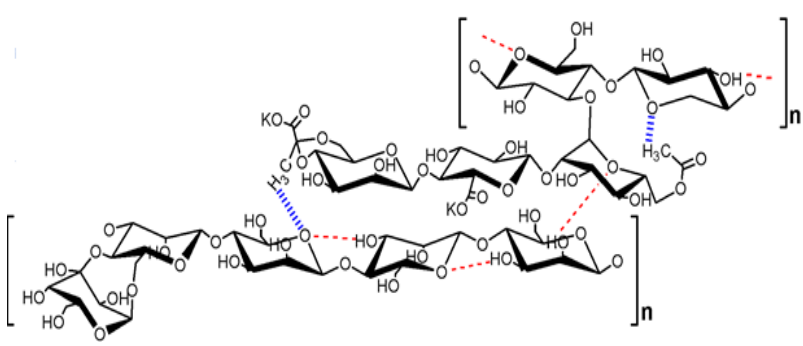

Figure 4. Co-gelation mechanism between xanthan and galactomannan. The dotted lines represent hydrogen bonding and broken lines van der Waals forces of attraction. As the tertiary structure of the xanthan molecule may keep a single stranded helix, its side-chains are inserted into the adjacent, unsubstituted segments of the backbone of the galactomannan molecule. A molecule of xanthan may combine with two or more molecules of galactomannan, the ratio depending on the favored conformation in aqueous solution. As the side-chains of the native and depyruvated xanthan molecules are somewhat rigid because of the intramolecular associations contributed by acetyl group and OH-3 of D-glucosyl residue, an incomplete interaction exist in part and greater interaction may result from deacetylation.

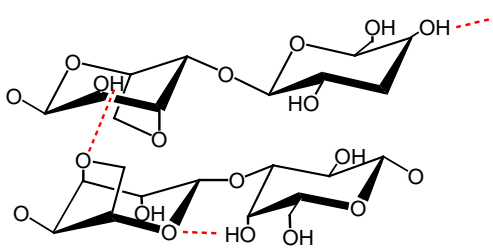

Figure 5. Gelation mechanism of agarose (agar). The dotted lines represent hydrogen bonding. 
gelation up to a high temperature $\left(60^{\circ} \mathrm{C}\right)$, and their periphery is surrounded by hydrophobic carbon and hydrogen atoms, which are required to play a role in the hydrophobic effect. This hydrophobic effect leads to hydrogen bonding that easily occurs within water molecules because of a decrease in entropy. Therefore, hydrogen bonding also takes place easily with water molecules on the outside of the polymer helices (Figure 6). The mode of intra- and inter-molecular hydrogen bonding of agarose molecules has been supported by ${ }^{1} \mathrm{H}$ - and ${ }^{13} \mathrm{C}-\mathrm{NMR}$ spectroscopy [35].

On the basis of this consideration, the agarose molecules play a dominant role in the center of tetrahedral cavities (cages) that are occupied by water molecules. This arrangement is similar to a tetrahedral ice-like structure and should lead to a cooperative effect. This effect stabilizes extended regions of the ice-like water that is hydrogen bonding on the surface of the polymer molecules. On the surface of the polymer molecules, ring oxygen and hydroxyl groups participate in hydrogen bonding with water molecules, and more extended ice-like hydrogen bonding with water molecules achieved to form a cluster. Namely, polysaccharide gel formation corresponds to a total of the cluster.

Accordingly, the gel-forming polysaccharides molecules, as mentioned above, adopt single, double, or multi-stranded conformations involving intra- and inter-molecular associations and play a role in the center of the tetrahedral cavities leading and stabilizing the extended regions of ice-like water molecules with hydrogen bonding, and resulting in gelation. On the contrary, many other non-gelling polysaccharides, such as welan gum [12,

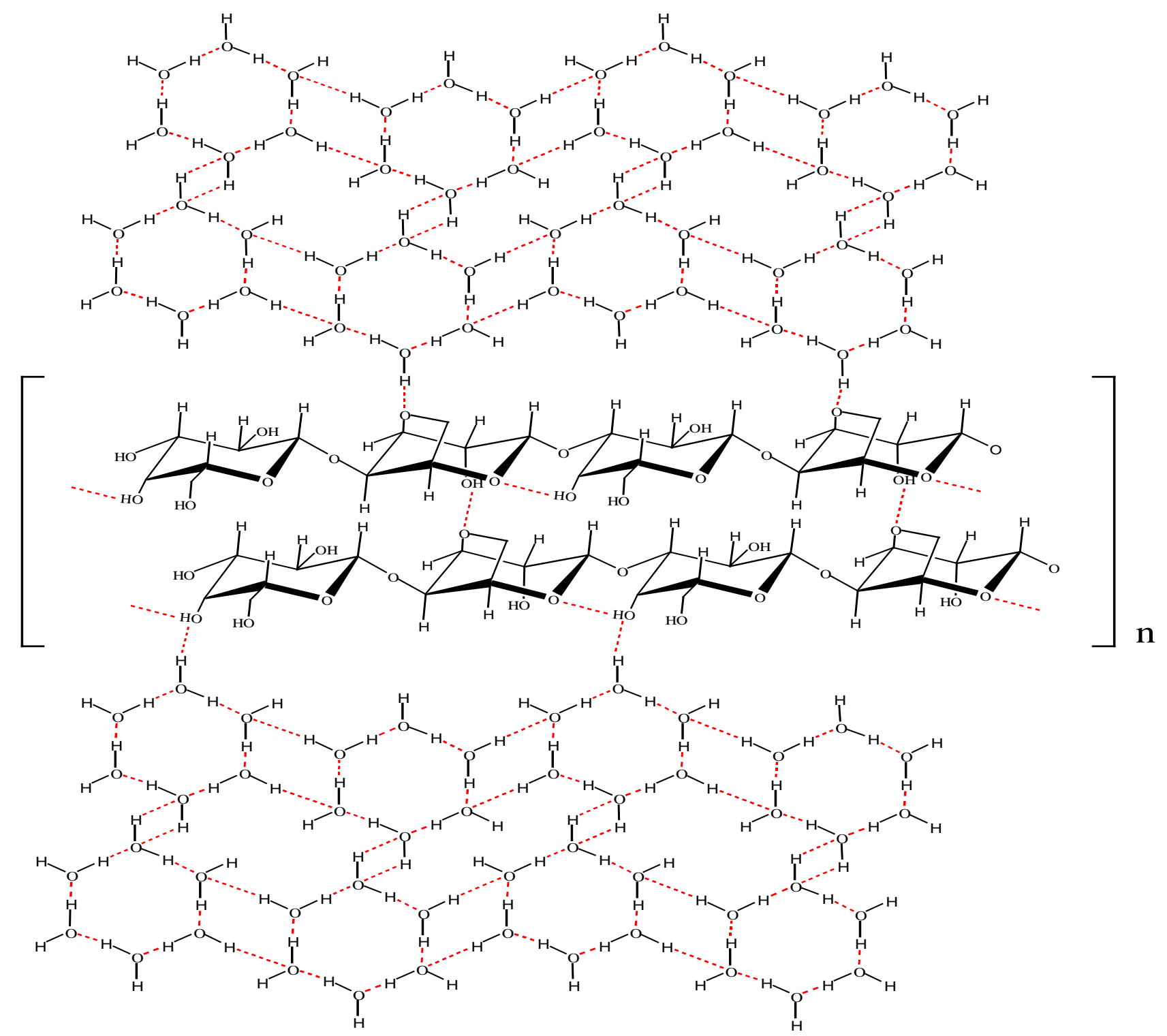

Figure 6. Gelling agarose in water molecules. The dotted lines represent hydrogen bonding. 
13], rhamsan gum [13,14], S-657 gum [15], S-88 gum [16], and schizophyllan [17], are free from intermolecular associations caused by the large kinetic energy and flexibility of the branched glycosidic linkages even involving intramolecular associations. These polysaccharides show the thermal stability for viscosity and dynamic viscoelasticity due to formation of intramolecular associations in aqueous solution.

Though many investigations concerning the gelling properties of the polysaccharides have been performed to determine the structure-function relationship, to the best of our knowledge, no other research group [18] has discussed what occurs with the water molecules at the molecular levels.

\section{Principles of Starch Gelatinization and Retrogradation}

Starch is the most common carbohydrate in human diets and used as gelling (pasting) and thickening agents in foods, paper making and some other industries. The physicochemical properties of starch obtained from different botanical sources vary due to difference in their amylose content, grain size and chain length distribution of amylopectin molecules.

We discuss the principle of gelation, and the gelatinization and retrogradation processes of amylose, amylopectin and starch molecules.

\subsection{Gelation and Retrogradation Mechanism of Amylose}

Amylose is a linear polysaccharide composed of 1,4linked $\alpha$-D-glucopyranosyl residues by definition, but the actual specimens that are isolated and purified from starch, include small amount of branched molecules [36].

After aqueous suspensions of amylose are heated at high temperatures $\left(120^{\circ} \mathrm{C}-140^{\circ} \mathrm{C}\right)$ and later cooled, amylose produces a gel. There are two stepwise conformational transition in amylose molecules under shearing force over a temperature range of $15^{\circ} \mathrm{C}-25^{\circ} \mathrm{C}$ and of $80^{\circ} \mathrm{C}-90^{\circ} \mathrm{C}$, respectively. The results indicated that there are two stepwise conformational transitions in amylose molecules. We proposed a gelation mechanism of amylose in aqueous solution as illustrated in Figure 7 [6]. The mechanism was suggested from the results that have been concluded by X-ray analysis at the solid state by Perez and Vergelati [37]. Intramolecular hydrogen bonding may occur between the OH-6 and the adjacent hemiacetal oxygen atom of the D-glucopyranosyl residues. In addition, intermolecular hydrogen bonding takes place between the $\mathrm{OH}-2$ and the adjacent O-6 of the D-glucopyranosyl residues on different molecules.

Amylose molecules that are at a concentration of $1.0 \%$ $(\mathrm{w} / \mathrm{v})$ in aqueous solution changed into a gel immediately under weak mechanical stimulation, which is caused by a cage effect that occur even at room temperature $\left(15^{\circ} \mathrm{C}\right.$ $20^{\circ} \mathrm{C}$ ).

The amylose molecules are notoriously unstable, and retrogradation results in increase of turbidity and eventual precipitation. Consequently, the retrogradation occurs because the amylose molecules shrink, which is caused by a decrease in kinetic energy and Brownian motion of the polymer and water molecules. This shrinkage results in new formation of intra- and intermolecular hydrogen bonding between both the hemiacetal oxygen atom and the adjacent OH-6 of the D-glucopyranosyl residues, and the O-6 and $\mathrm{OH}-2$ of D-glycopyranosyl residues on different molecules, as presented in Figure 8. Much more intense intra- and inter-molecular hydrogen bonding leads to precipitation of the amylose molecules in aqueous media [7].

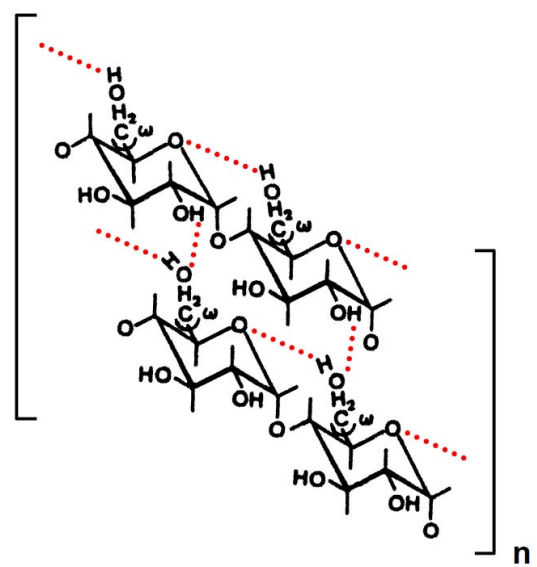

Figure 7. Gelation mechanism of amylose. The dotted lines represent hydrogen bonding.

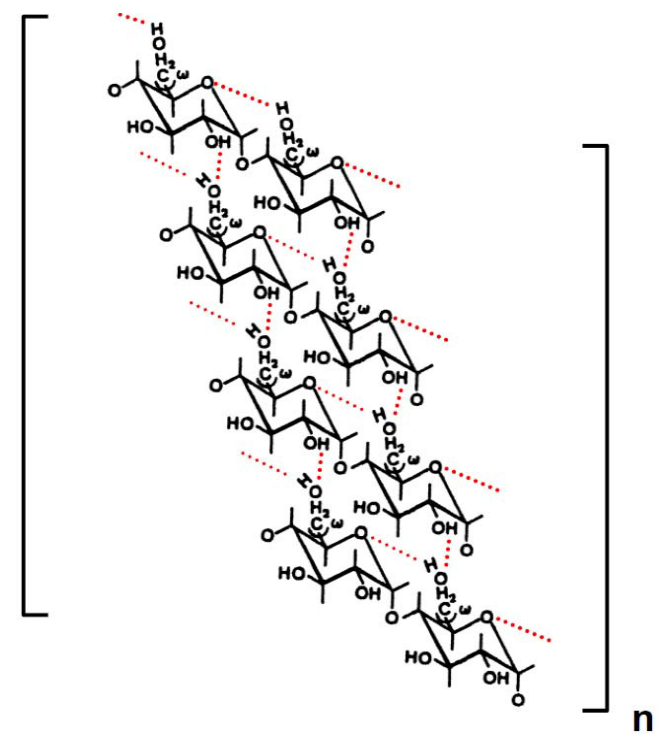

Figure 8. Retrogradation mechanism of amylose. The dotted lines represent hydrogen bonding. 


\subsection{Molecular Origin for Thermal Stability of Rice Amylopectin and Its Retrogradation Mechanism}

The most widely accepted model for the amylopectin molecule which is a branched macromolecule composed of 1,4-(94\% - 96\%) and 1,6-linked (4\% - 6\%) $\alpha$-D-glucopyranosyl residues, is the cluster model [38-40]. As reported by proceeding papers [41-43], rice amylopectin solution does not gel even at high concentration (6.0\%) and low temperature $\left(0^{\circ} \mathrm{C}\right)$. However, the elastic modulus of the solution showed high value and kept a constant during increase in temperature even at high temperature $\left(80^{\circ} \mathrm{C}\right)$. Thus, we concluded that the molecules are involved in either intramolecular hydrogen bonding or van der Waals forces of attraction between either the OH-6 and the adjacent hemiacetal oxygen atom or between O-6 and anomeric hydrogen of the adjacent D-glucopyranosyl residue, as illustrated in Figures 9 and 10 [41-43]. The methylene group of C-6 of the D-glucopyranosyl residue might also partially participate in the van der Waals forces of attraction with the hemiacetal oxygen atom. Intramolecular hydrogen bonding and van der Waals forces of attraction play dominant roles in the thermal stability of rice amylopectin molecules in solutions, leading to a stable viscosity and elastic modulus.

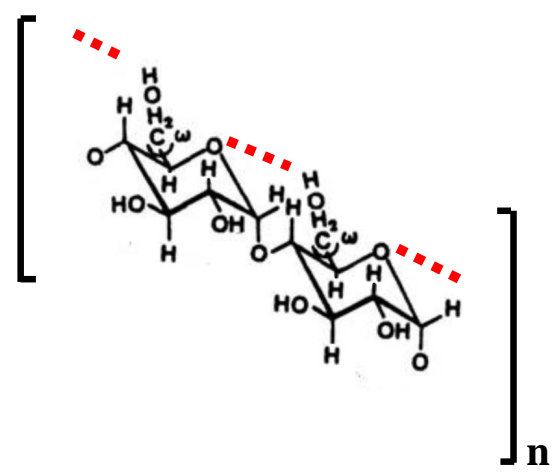

Figure 9. Intramolecular hydrogen bonding of amylosepectin. The dotted lines represent hydrogen bonding.

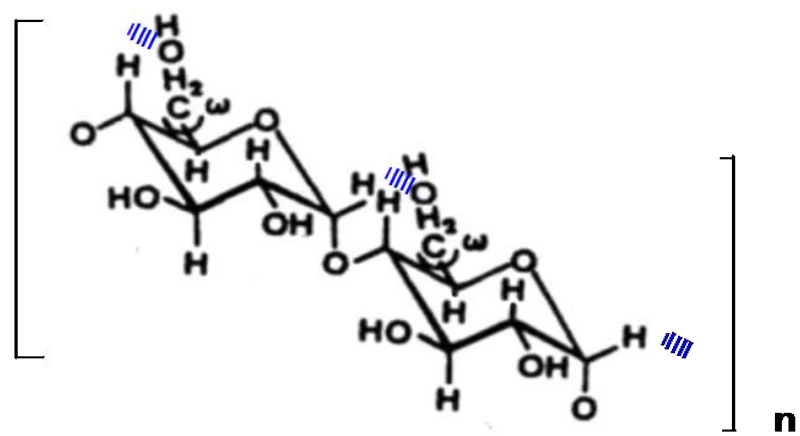

Figure 10. Intramolecular van der Waals forces of attraction of amylopectin. The broken lines represent van der Waals forces of attraction.
In contrast, wheat amylopectin molecules $[44,45]$ exhibit a lower elastic modulus than that of rice amylopectins [41-43]. Viscosity and elastic modulus decrease gradually with an increase in temperature and is low at high temperature $\left(80^{\circ} \mathrm{C}\right)$. However, the elastic modulus of the polymer solution that is increased to $4.0 \mathrm{M}$ urea and 0.05 $\mathrm{M} \mathrm{NaOH}$, respectively, suggests that there are insoluble fragments, where intermolecular hydrogen bonding take place of polymer molecules in aqueous solutions is taking place. Thus, the structure of wheat amylopectin molecules seems to differ from that of rice amylopectin.

The high solubility and thermal stability of rice amylopectin molecules may be attributed to large number of branching short side-chains (A) on long chains (B2-B3). Consequently, the short side-chains (A) of rice amylopectin molecules prevent intermolecular hydrogen bonding and are likely to dissolve easily in water compared with those of wheat amylopectin molecules. The branching structure of the wheat and rice amylopectin molecules corresponds to the cluster model in which highly branching side-chains (A and B1) and less branching regions are involved along long chains (B2-3). Insoluble fragments of wheat amylopectin molecules also attributed to an involvement of a little large number of super-long chains, $2 \%-4 \%[46,47]$ than those of rice amylopectin molecules, $1.5 \%$ [48,49]. The super-long chains without side chains, like amylose, on amylopectin molecules are liable to associate with neighboring long chains of polymer molecules with hydrogen bonding resulted in formation of the insoluble fragments in aqueous solution.

In their daily life, Japanese people often eat the traditional rice cakes (amylopectin: called Mochi). The rice cake has soft texture after preparation (water content $55 \%$ - 60\%). Specifically, the Japanese make it a custom to decorate Kagami-mochi (rice cake) for ten or more days at the beginning new year. The rice cake contains approximately $45 \%$ - 50\% water. After decorating the rice cake, it cannot be broken even with hammer. The hardness of the rice cake is due to the formation of hydrogen bonding between polymer-polymer, polymerwater, and water-water molecules. The hydrogen bonding occurs due to a decrease in kinetic energy and Brownian motion of the polymer and water molecules during storage (Figure 11). The mochi, however, has been recovered original soft texture by heating due to increase in kinetic energy which leads to break down the tetrahedral hydrogen bonding between polymer-polymer, polymerwater and water-water molecules.

\subsection{Gelatinization and Retrogradaion Mechanism of Starch}

Starch gelatinization in water is a process on break down of the intermolecular association between amylose and 


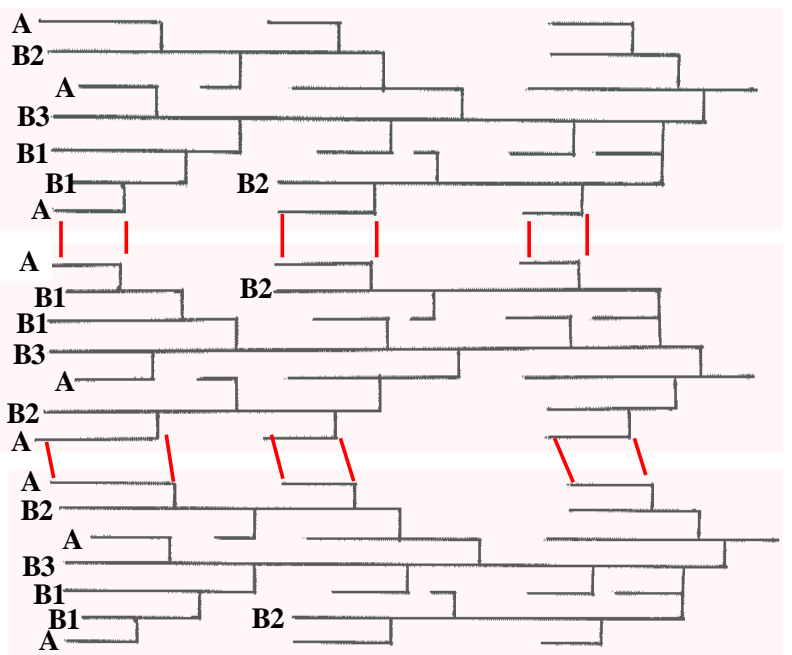

Figure 11. Association sites shown in red lines between amylopectin molecules (Japanese traditional rice cake: Mochi). After storage for about $\mathbf{1 0}$ days, much more intense intermolecular association between amylopectin molecules takes place and water molecules also take part in the association due to decrease in kinetic energy and Brownian motion.

amylopectin molecules at solid state (granules) with heating. After dissolving the starch molecules on heating, it shows high viscous solution and changes into strong gelling (retrogradation) state on storage for long times.

We have proposed gelatinization mechanism of rice [48,49], potato [50], and wheat starch [51,52]. The intermolecular association might take place between the O-6 of amylose and the OH-2 of amylopectin molecules due to hydrogen bonding, as illustrated in Figure 12. The short amylopectin chains (A and B1) take part in intermolecular associations. Intermolecular hydrogen bonding between amylose and amylopectin molecules is thermally stable. The mechanism has been suggested for cogelation mechanism between xanthan gum and galactomannan, and for kojac glucomannnan, where trisaccharide side-chains of the former molecules contributed, as depicted in Figure 4. Two or more short side chains (A and B1) of amylopectin molecules may associate with an amylose molecule, because rice, potato and wheat starch consists of $19 \%-23 \%$ amylose and $81 \%$ - $77 \%$ amylopectin.

The elastic modulus of rice [48,49] and potato [50] starch decreased in a $4.0 \mathrm{M}$ urea and $0.05 \mathrm{M} \mathrm{NaOH}$ solution, respectively, even at low temperature, but maintain a constant value during the increase in temperature. On the contrary, for wheat starch, the elastic modulus increases and stays at a constant value with increasing temperature in the same urea and $\mathrm{NaOH}$ solution, respectively [51,52]. The increase in elastic modulus has been caused by dissolving amylose and amylopectin molecules completely in $4 \mathrm{M}$ urea and $0.05 \mathrm{M} \mathrm{NaOH}$ solution.

The elastic modulus increases when the rice [53], po- tato [50] and wheat [51] starch solutions are stored at 25 and $4^{\circ} \mathrm{C}$ for $24 \mathrm{~h}$, retrogradation occurs after formation of new intermolecular hydrogen bonding. Thus, we concluded that another intermolecular hydrogen bond might form between the $\mathrm{OH}-2$ of a D-glucopyranosyl residue of the amylose and the O-6 of a D-glucopyranosyl residue of short side-chain (A and B1) of the amylopectin molecules, as illustrated in Figure 13.

After saturation of intermolecular hydrogen bonding between amylose and amylopectin molecules, an intermolecular association also takes places between amylopectin molecules due to hydrogen bonding. This bonding is caused by a decrease in Brownian motion and kinetic energy of amylopectin and water molecules during storage. At the final stage, the side-by-side association between the O-3 and the OH-3 of D-glucopyranosyl

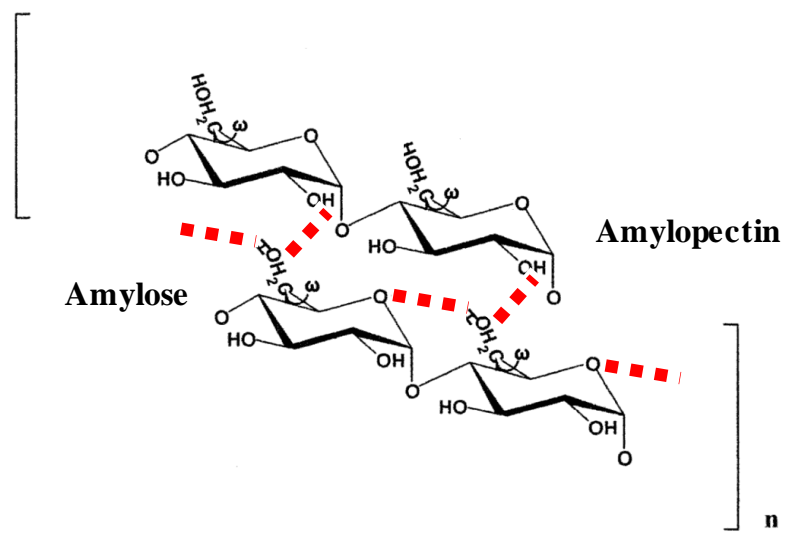

Figure 12. Gelatninzation mechanism of starch. The dotted lines represent hydrogen bonding.

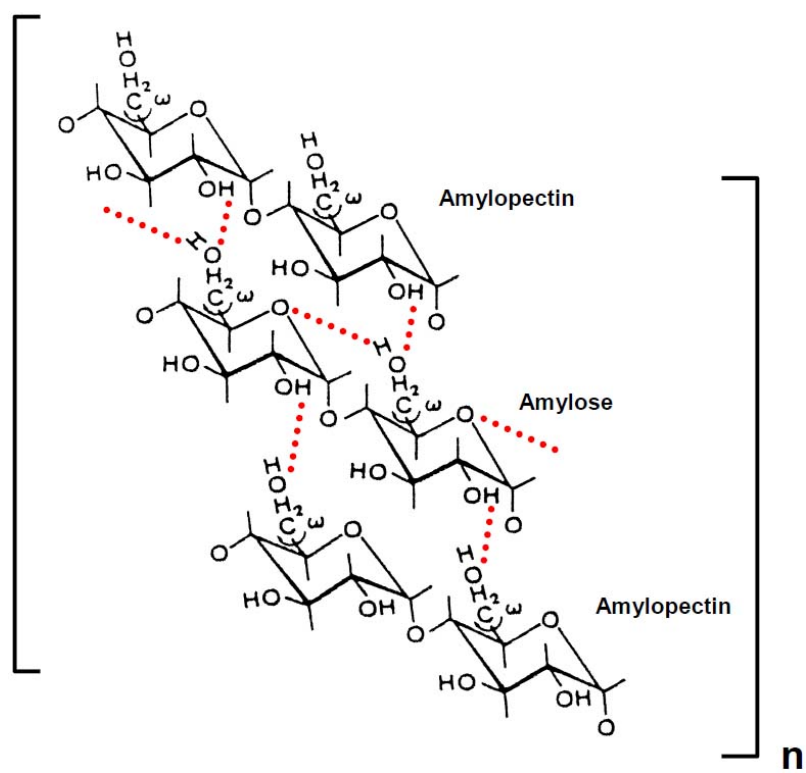

Figure 13. Retrogradation mechanism of rice, potato and wheat starches. The dotted lines represent hydrogen bonding. 
residues on different amylopectin molecules may also take place (Figure 14).

\section{Principle of Starch Gelatinization and Retrogradation}

The water molecule $\left(\mathrm{H}_{2} \mathrm{O}\right)$ can participate in four hydrogen bonds, in which two bonding involve the two hydrogens and the lone pair of electrons of the oxygen and the hydrogen of two neighboring water molecules. This tetrahedrally directed bonding of water molecules, however, is partially involved in gelatinized starch solutions due to the higher kinetic energy of the short side chains (A and B1) of amylopectin molecules.

Thus, we demonstrate intermolecular interaction between starch and water, and between water molecules in Figure 15. The starch molecules (4\%) that adopt a hexagonal conformation involving intra- and inter-molecular

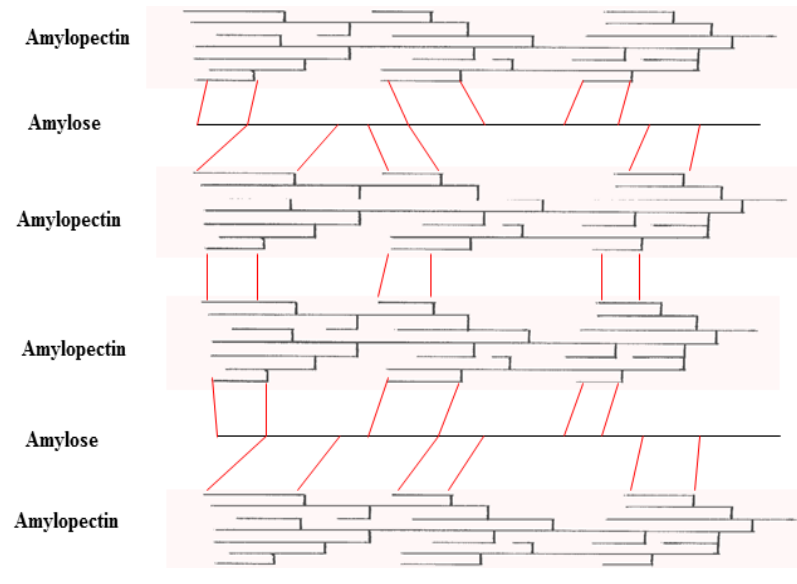

Figure 14. Association sites shown with red lines between amylose and amylopectin molecules of rice starch. Two or more short side-chains (A or B1) of amylopectin molecules take part in the interaction with an amylose molecule.

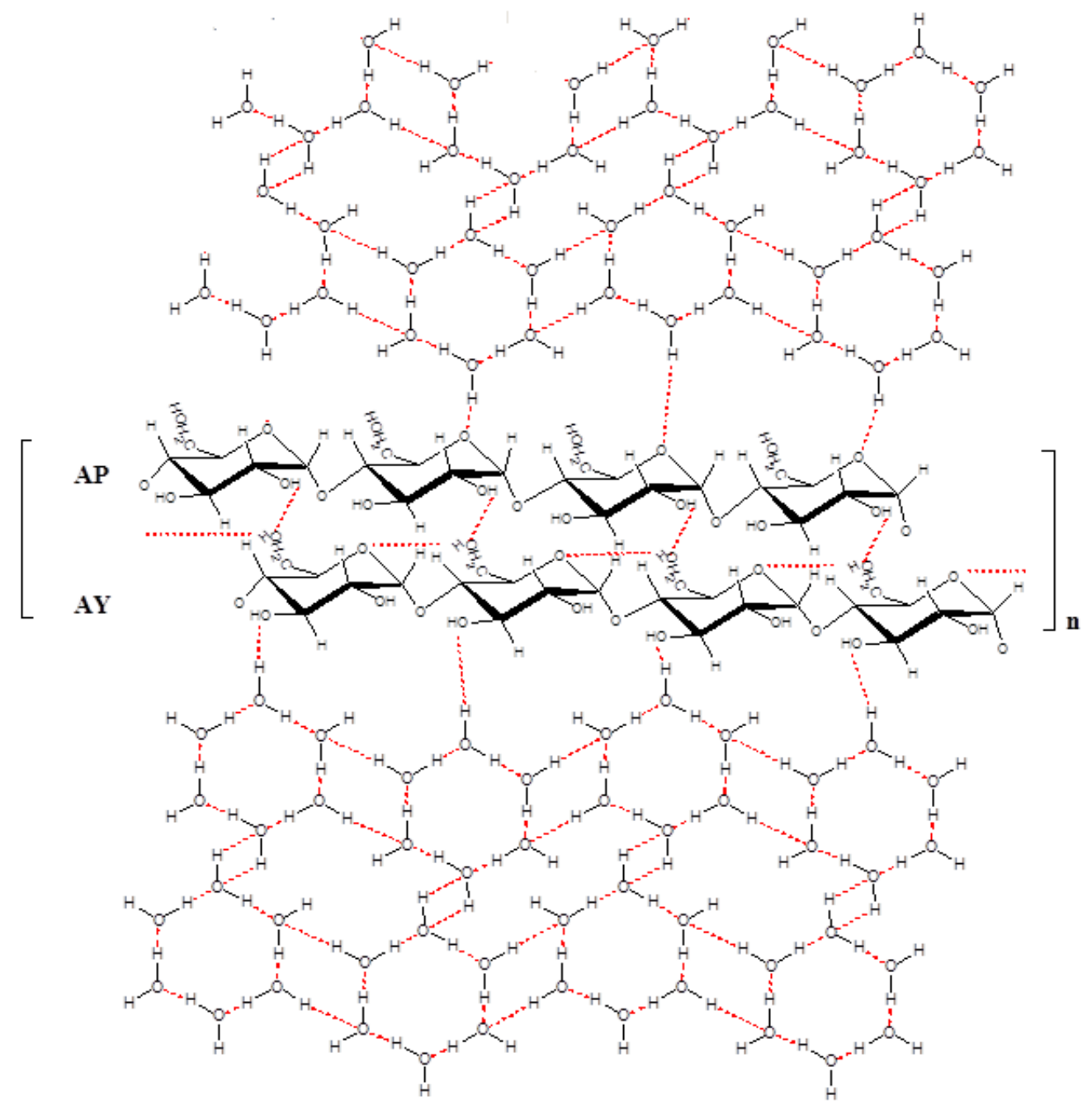

Figure 15. Possible gelatinization mechanism including water molecules of rice, potato, and wheat starches. The dotted red lines represent hydrogen bonding. AY, Amylose; AP, short chain (A or B1) of amylopectin molecules. Water molecules are associated in part with the A or B1 chain of amylopectin molecules caused by a little high kinetic energy of the short side chains. 
hydrogen bonding changed approximately large number of the liquid water molecules into a network of tetrahedrally hydrogen-bonded water molecules in part, even at room temperature, which results in gelatinization. The hemiacetal oxygen $(>0)$ and hydroxyl groups of the starch molecules may be responsible for arranging a network of tetrahedrally hydrogen-bonded water molecules. This network is formed by a cage effect leading to the lower energy state of the lone pair of electrons on the water molecules and extends into hydrogen bonding between water molecules even at room temperature, although the lowest energy has been established at a temperature of $0^{\circ} \mathrm{C}$ pure water molecules.
For the retrogradation process, much more intense intermolecular hydrogen bonding takes place between amylose and amylopectin and between amylopectin molecules, as illustrated in Figure 16. After saturation of intermolecular hydrogen bonding between amylose and amylopectin molecules, an intermolecular association may also take place between amylopectin molecules due to hydrogen bonding. This bonding is caused by a decrease of kinetic energy and Brownian motion of amylopectin and water molecules during storage. At this stage, sideby-side association between the $\mathrm{O}-3$ and the $\mathrm{OH}-3$ of D-glucopyranosyl residues on different amylopectin molecules may also take place (Figure 14).

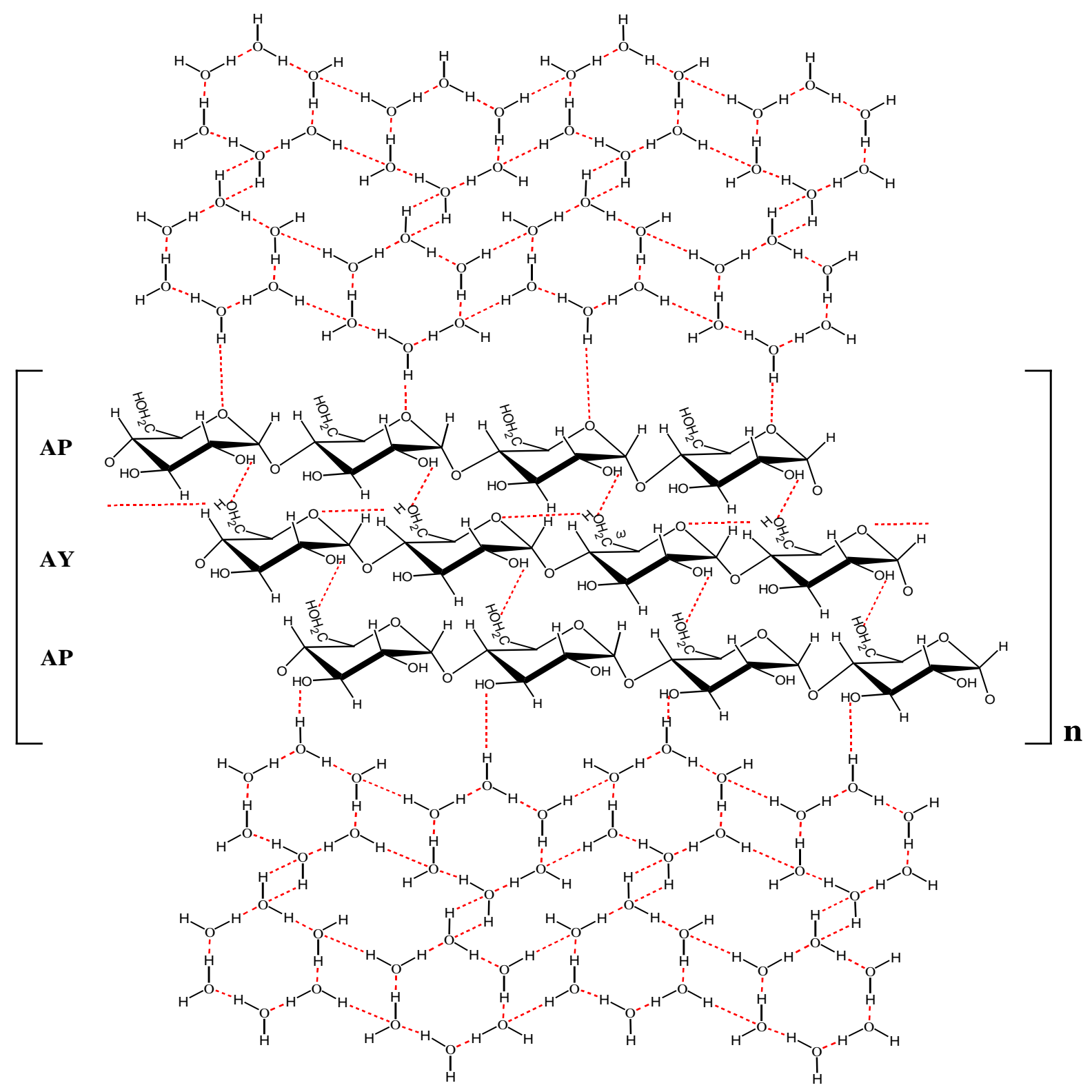

Figure 16. Retrogradation mechanism including water molecules of rice, potato and wheat starches. The dotted lines represent hydrogen bonding. AY, Amylose; AP, short chain (A or B1) of amylopectin molecules. 


\section{Conclusions}

The amylose molecules associate within and among polymer molecules resulting in precipitation. Such tendency, homogeneous association, also occurs among amylopectin molecules, but it takes long time due to presence of short side chains (A and B1) that was caused by their high kinetic energy. The starch retrogradation is a process that a gelatinized solution is cooled for a long time, it changes into gel (thicken) and rearranges itself again to a crystalline structure (granule). Consequently, the starch molecules take minimum volumes (granules) not only in solution, but also in plants.

It is well known that cellulose molecules (1,4-linked $\beta$-D-glucan) associate with hydrogen bonding where hydroxyl groups at C-2 and C-6 of D-glucopyranosyl residues contribute [54,55]. The contribution of hydroxyl groups at C-2 and C-6 of D-glucopyranosyl residues on gelation, gelatinization and retrogradation processes on amylose, amylopectin and starch molecules was suggested from crystal structure of cellulose [54], amylose and amylopectin [37] molecules proposed by X-ray analysis at solid states.

Hydrogen-bonded water molecules in a tetrahedral configuration might be partially involved in the gelatinization processes in starch solutions which is caused by the high kinetic energy of short side chains (A and B1) of amylopectin molecules in aqueous solutions. The starch gelatinization and retrogradation processes together with the polysaccharide gelation processes as discussed above [18] provide important suggestions in academic and industrial fields.

Though many investigations include not only starch gelatinization and retrogradaion, but also the gelling properties of the polysaccharides which have been undertaken to elucidate the structure-function relationship. No other researchers have established mechanism at the molecular level. There is a reasonable consistency in our investigations. Thus, rheological analysis is one of significant methods for understanding the structure-function relationship of polysaccharides in aqueous media. We expect that the era of polysaccharides gels will be arriving in the 21st century, with such processes as starch gelatinization and retrogradation, being applied not only from the food industry, but also in a variety of products, such as cosmetics, pharmaceuticals and chemicals.

\section{REFERENCES}

[1] M. Tako and S. Nakamura, "Indicative Evidence for a Conformational Transition in $\kappa$-Carrageenan from Studies of Viscosity-Shear Rate Dependence," Carbohydrate Research, Vol. 155, No. 1, 1986, pp. 200-205. http://dx.doi.org/10.1016/S0008-6215(00)90146-0

[2] M. Tako and S. Nakamura, "Synergistic Interaction be- tween $\kappa$-Carrageenan and Locust Bean Gum in Aqueous Media," Agricultural and Biological Chemistry, Vol. 50, No. 11, 1986, pp. 2817-2822. http://dx.doi.org/10.1271/bbb1961.50.2817

[3] M. Tako, S. Nakamura and Y. Kohda, "Indicative Evidence for a Conformational Transition in $t$-Carrageenan," Carbohydrate Research, Vol. 161, No. 2, 1987, pp. 247253. http://dx.doi.org/10.1016/S0008-6215(00)90081-8

[4] M. Tako and S. Nakamura, "Gelation Mechanism of Agarose,” Carbohydrate Research, Vol. 180, No. 2, 1988, pp. 277-284.

http://dx.doi.org/10.1016/0008-6215(88)80084-3

[5] M. Tako, A. Sakae and S. Nakamura, "Rheological Properties of Gellan Gum in Aqueous Media,” Agricultural Biological Chemistry, Vol. 53, No. 3, 1989, pp. 771-776. http://dx.doi.org/10.1271/bbb1961.53.771

[6] M. Tako and S. Hizukuri, "Evidence for Conformational Transition in Amylose," Journal of Carbohydrate Chemistry, Vol. 14, No. 4-5, 1995, pp. 613-622.

http://dx.doi.org/10.1080/07328309508005362

[7] Y. Tamaki, T. Konishi and M. Tako, "Gelation and Retrogradation Mechanism of Wheat Amylose," Materials, Vol. 4, No. 10, 2011, pp. 1763-1775. http://dx.doi.org/10.3390/ma4101763

[8] M. Tako and I. Hanashiro, "Evidence for a Conformational Transition in Curdlan,” Polymer Gels \& Networks, Vol. 5, No. 2, 1997, pp. 241-250. http://dx.doi.org/10.1016/S0966-7822(96)00036-6

[9] M. Tako and Y. Kohda, "Calcium Induced Association Characteristics of Alginate,” Journal of Applied Glycoscience, Vol. 44, No. 2, 1997, pp. 153-159.

[10] M. Tako, S. Tohma, T. Taira and M. Ishihara, "Gelation Mechanism of Deacetylated Rhamsan Gum,” Carbohydrate Polymers, Vol. 54, No. 3, 2003, pp. 279-285. http://dx.doi.org/10.1016/S0144-8617(03)00029-8

[11] M. Tako, T. Teruya, Y. Tamaki and T. Konishi, "Molecular Origin for Rheological Characteristics of Native Gellan Gum,” Colloid and Polymer Science, Vol. 287, No. 12, 2009, pp. 1445-1454. http://dx.doi.org/10.1007/s00396-009-2112-2

[12] M. Tako and M. Kiriaki, "Rheological Properties of Welan Gum in Aqueous Medi,” Agricultural Biological Chemistry, Vol. 54, No. 12, 1990, pp. 3079-3084. http://dx.doi.org/10.1271/bbb1961.54.3079

[13] M. Tako, "Molecular Origin for the Thermal Stability of Welan and Rhamsan Gum,” In: M. Yalpani, Ed., Carbohydrate and Carbohydrate Polymers, ATL Press, Inc. Science and Publishers, Mount Prospect, 1993, pp. 205215.

[14] M. Tako, "Molecular Origin for Thermal Stability of Rhamsan Gum in Aqueous Media,” Bioscience, Biotechnology, and Biochemistry, Vol. 57, No. 7, 1993, pp. 11821184. http://dx.doi.org/10.1271/bbb.57.1182

[15] M. Tako, "Molecular Origin for Thermal Stability of S-657 Gum Produced by Xanthomonas ATCC53159," Polymer Gels \& Networks, Vol. 2, No. 2, 1994, pp. 91104. http://dx.doi.org/10.1016/0966-7822(94)90029-9

[16] M. Tako and H. Tamaki, "Molecular Origin for Thermal 
Stability of S-88 Gum Produced by Pseudomonas ATCC31554,” Polymer Journal, Vol. 37, No. 7, 2005, pp. 498-505. http://dx.doi.org/10.1295/polymj.37.498

[17] M, Tako, "Molecular Origin for Thermal Stability of Schizophyllan,” Polymer Gels \& Networks, Vo. 4, No. 4, 1996, pp. 303-313. http://dx.doi.org/10.1016/0966-7822(96)00016-0

[18] M. Tako, "Structural Principle of Polysaccharide Gels," Journal of Applied Glycoscience, Vol. 47, No. 1, 2000, pp. 49-53. http://dx.doi.org/10.5458/jag.47.49

[19] M. Tako, T. Nagahama and D. Nomura,” Non-Newtonian Behavior and Dynamic Viscoelasticity of Xanthan Gum,” Nippon Nogeikagaku Kaishi, Vol. 51, No. 8, 1977, pp. 513-517. http://dx.doi.org/10.1271/nogeikagaku1924.51.8 513

[20] P.-E. Jansson, L. Kenne and B. Lindberg, "Structure of the Extracellular Polysaccharide from Xanthomonas campestris,” Carbohydrate Research, Vol. 45, No. 1, 1975, pp. 275-282.

http://dx.doi.org/10.1016/S0008-6215(00)85885-1

[21] M. Tako and S. Nakamura, "Rheological Properties of Deacetylated Xantham Gum in Aqueous Media,” Agricultural Biological Chemistry, Vol. 48, No. 12, 1984, pp. 2987-2993. http://dx.doi.org/10.1271/bbb1961.48.2987

[22] M. Tako and S. Nakamura, "Rheological Properties of Ca salt of Xanthan Gum in Aqueous Media,” Agricultural Biological Chemistry, Vol. 51, No. 11, 1987, pp. 29192923. http://dx.doi.org/10.1271/bbb1961.51.2919

[23] M. Tako and S. Nakamura, "Rheological Properties of Depyruvated Xanthan Gum in Aqueous Media,” Agricultural Biological Chemistry, Vol. 52, No. 6, 1988, pp. 1585-1586.

[24] M. Tako and S. Nakamura, "Evidence for Intramolecular Associations in Xanthan Molecules in Aqueous Media," Agricultural Biological Chemistry, Vol. 53, No. 7, 1989, pp. 1941-1946. http://dx.doi.org/10.1271/bbb1961.53.1941

[25] M. Tako, "Molecular Origin for Rheological Characteristics of Xanthan Gum,” ACS Symposium Series, Vol. 489, 1992, pp. 268-281. http://dx.doi.org/10.1021/bk-1992-0489.ch017

[26] M. Tako, A. Asato and S. Nakamura, "Rheological Aspects of Intermolecular Interaction between Xanthan and Locust Bean Gum in Aqueous Media,” Agricultural Biological Chemistry, Vol. 48, No. 12, 1984, pp. 2995-3000. http://dx.doi.org/10.1271/bbb1961.48.2995

[27] M. Tako and S. Nakamura, "Synergistic Interaction between Xanthan and D-Galacto-D-Mannan," FEBS Letters, Vol. 204, No. 1, 1986, pp. 33-36. http://dx.doi.org/10.1016/0014-5793(86)81382-5

[28] M. Tako, "Synergistic Interaction between Deacylated Xanthan and Galactomannan," Journal of Carbohydrate Chemistry, Vol. 10, No. 4, 1991, pp. 619-633. http://dx.doi.org/10.1080/07328309108543936

[29] M. Tako and S. Nakamura, "Synergistic Interaction between Xanthan and Guar Gum," Carbohydrate Research, Vol. 138, No. 1, 1985, pp. 207-213. http://dx.doi.org/10.1016/0008-6215(85)85104-1
[30] M. Tako, "Synergistic Interaction between Xanthan and Tara-Bean Gum,” Carbohydrate Polymers, Vol. 16, No. 3, 1991, pp. 227-239. http://dx.doi.org/10.1016/0144-8617(91)90111-O

[31] P. Pakdee, M. Tako, T. Yokohari, K. Kinjyo, F. Hongo and S. Yaga, "Synergistic Interaction between Xanthan and Galactomannan Isolated from Leucaena leucocephala de Wit,” Journal of Applied Glycoscience, Vol. 42, No. 2, 1995, pp. 105-113.

[32] M. Tako, T. Teruya, Y. Tamaki and K. Okawa, "CoGelation Mechanism of Xanthan and Galactomannan,” Colloid and Polymer Science, Vol. 288, No. 10-11, 2010, pp. 1161-1166.

http://dx.doi.org/10.1007/s00396-010-2242-6

[33] M. Tako, "Synergistic Interaction between Xanthan and Konjac Glucomannan in Aqueous Media,” Bioscience, Biotechnology, and Biochemistry, Vol. 56, No. 8, 1992, pp. 1188-1192. http://dx.doi.org/10.1271/bbb.56.1188

[34] M. Tako, "Binding Sites for D-Mannose-Specific Interaction between Xanthan and Galactomannan, and Glucomannan," Colloids and Surfaces B: Biointerfaces, Vol. 1, No. 2, 1993, pp. 125-131.

http://dx.doi.org/10.1016/0927-7765(93)80043-X

[35] A. Gamini, R., Toffanin, E. Murano and R. Rizzo, "Hydrogen Bonding and Conformation of Agarose in Methyl Sulfoxide and Aqueous Solutions Investigated by ${ }^{1} \mathrm{H}$ and ${ }^{13}$ C NMR Spectroscopy," Carbohydrate Research, Vol. 304, No. 3-4, 1997, pp. 293-302. http://dx.doi.org/10.1016/S0008-6215(97)00232-2

[36] Y. Takeda, S. Tomooka and S. Hizukuri, "Structure of Branched and Linear Molecules of Amylose," Carbohydrate Research, Vol. 246, No. 1, 1993, pp. 267-272. http://dx.doi.org/10.1016/0008-6215(93)84038-8

[37] S. Perez and C. Vergelati, "Solid State and Solution Features of Amylose and Amylose Fragments," Polymer Bulletin, Vol. 17, No. 2, 1987, pp. 141-148. http://dx.doi.org/10.1007/BF00256880

[38] J. Nikuni, “Starch and Cookery,” Chyouri Kagaku, Vol. 2, No. 1, 1969, pp. 6-10.

[39] D. French,” Fine Structure and Relationship to the Organization of Starch Granules," Journal of Japanese Society of Starch Science, Vol. 19, No. 1, 1972, pp. 8-25. http://dx.doi.org/10.5458/jag1972.19.8

[40] S. Hizukuri, "Polymodal Distribution of the Chain Length of Amylopectin, and Its Significant," Carbohydrate Research, Vol. 147, No. 2, 1986, pp. 342-347. http://dx.doi.org/10.1016/S0008-6215(00)90643-8

[41] M. Tako, "Molecular Origin for Thermal Stability of Waxy Rice Starch,” Staerke/Starch, Vol. 48, 1999, pp. 414-417.

[42] M. Tako and S. Hizukuri, "Molecular Origin for the Thermal Stability of Rice Amylopectin,” Journal of Carbohydrate Chemistry, Vol. 16, No. 4-5, 1997, pp. 655-666. http://dx.doi.org/10.1080/07328309708007343

[43] M. Tako and S. Hizukuri, "Molecular Origin for Thermal Stability of Koshihikari Rice Amylopectin,” Food Research International, Vol. 33, No. 1, 2000, pp. 35-40. http://dx.doi.org/10.1016/S0963-9969(00)00021-1 
[44] M. Tako and S. Hizukuri, "Rheological Properties of Wheat (Halberd) Amylopectin,” Staerke/Starch, Vol. 55, No. 8, 2003, pp. 345-349.

[45] M. Tako, I. Hanashiro and S. Uechi, "Rheological Properties of Wheat Amylopectin," Science of Bulletin of Faculty of Agriculture, University of the Ryukyus, No. 51, 2004, pp. 139-143.

[46] K. Shibanuma, Y. Takeda and S. Hizukuri, "Molecular Structure of Some Wheat Starches," Carbohydrate Polymers, Vol. 25, No. 2, 1994, pp. 111-116. http://dx.doi.org/10.1016/0144-8617(94)90146-5

[47] K. Shibanuma, Y. Takeda and S. Hizukuri, "Molecular and Pasting Properties of Some Wheat Starches," Carbohydrate Polymers, Vol. 29, No. 3, 1996, pp. 253-261. http://dx.doi.org/10.1016/0144-8617(96)00026-4

[48] M. Tako and S. Hizukuri, "Gelatinization Mechanism of Rice Starch,” Journal of Carbohydrate Chemistry, Vol. 18, No. 5, 1999, pp. 573-584. http://dx.doi.org/10.1080/07328309908544020

[49] M. Tako, "Gelatinization Characteristics of Rice Starch," Journal of Applied Glycoscience, Vol. 47, No. 2, 2000, pp. 187-192. http://dx.doi.org/10.5458/jag.47.187

[50] M. Tako and S. Hizukuri, "Gelatinization Mechanism of Potato Starch,” Carbohydrate Polymers, Vol. 48, No. 4, 2003, pp. 397-401.
http://dx.doi.org/10.1016/S0144-8617(01)00287-9

[51] M. Tako, Y. Tamaki, T. Konishi, K. Shibanuma, I. Hanashiro and Y. Takeda," Gelatinization and Retrogradation Characteristics of Wheat (Rosella) Starch," Food Research International, Vol. 41, No. 8, 2008, pp. 797-802. http://dx.doi.org/10.1016/j.foodres.2008.07.002

[52] M. Tako, Y. Tamaki, T. Teruya, T. Konishi, K. Shibanuma, I. Hanashiro and Y. Takeda, "Gelatinization Characteristics of Halberd Wheat Starch," Staerke/Starch, Vol. 61, No. 5, 2009, pp. 275-281.

[53] M. Tako and S. Hizukuri, "Retrogradation Mechanism of Rice Starch,” Cereal Chemistry, Vol. 77, No. 4, 2000, pp. 473-477. http://dx.doi.org/10.1094/CCHEM.2000.77.4.473

[54] A. Sarko, J. Southwick and J. Hayashi, "Packing Analysis of Carbohydrates and Polysaccharides. 7. Crystal Structure of Cellulose III and Its Relationship to Other Cellulose Polymorphs,” Macromolecules, Vol. 9, No. 5, 1976, pp. 857-863. http://dx.doi.org/10.1021/ma60053a028

[55] Z. M. Ford, E. D. Stevens, G. P. Johnson and A. D. French, "Determining the Crystal Structure of Cellulose III by Modeling," Carbohydrate Research, Vol. 340, No. 5, 2005, pp. 827-833. http://dx.doi.org/10.1016/j.carres.2005.01.028 